\title{
ON MAPPINGS OF A UNIFORM SPACE ONTO ITSELF
}

BY

\section{ALBERT EDREI}

Let $E$ and $F$ be two uniform spaces $\left({ }^{1}\right)$ and denote by $\mathcal{F}(E, F)$ the set of all mappings of $E$ into $F$. We introduce, in $\mathcal{F}(E, F)$, the structure of uniform convergence so that families of mappings can also be considered as sets of points of the functional space $\mathcal{F}_{u}(E, F)$ (cf. [2, chap. 10, p. 5]).

We shall mainly study the special case $E=F$ and this will lead us to certain modifications and extensions of the notion of equicontinuity (Theorems 1, 2, 3 and their corollaries). However, we find it convenient to state our definitions in the general case, $E \neq F$, and also to investigate their more immediate implications (Properties I to V, Lemma 1, Theorem of Ascoli-Gottschalk).

1. Definitions. Let $\{U\}$ and $\{V\}$ be the filters of vicinities $\left({ }^{2}\right)$ which define, respectively, the structures of $E$ and $F$.

We say:

(i) that two mappings $h_{1}$ and $h_{2}$ differ by less than $V$ if

$$
h_{1}(x) \subset V\left(h_{2}(x)\right) \text { and } h_{2}(x) \subset V\left(h_{1}(x)\right) \text {, }
$$

for all $x[\in E]$;

(ii) that $h$ is $V$-continuous if there exists $U$ such that

$$
h(U(x)) \subset V(h(x))
$$

for all $c[\in E]$

(iii) that a finite set of mappings

$$
h_{1}, h_{2}, \cdots, h_{q} \text {, }
$$

is a $V$-net( $\left(^{3}\right)$ for the family $H$, if every function of $H$ differs, by less than $V$, from some member of (1);

(iv) that a family $H$ is almost smooth if, to every $V$, there corresponds a non-negative integer $n(V)$, such that all but $n(V)$ members of $H$ are $V$-continuous (if $n(V) \equiv 0$, every member of $H$ is uniformly continuous);

Received by the editors May 18, 1950.

(1) Throughout this paper, we use the basic concepts of the theory of uniform spaces as expounded in Bourbaki [2]. However, we need very few of Bourbaki's results. The mere definition of a uniform space and the notion of "structure of uniform convergence" should prove sufficient for the understanding of most results of this paper. Numbers in brackets refer to the references cited at the end of the paper.

${ }^{2}$ ) We translate "entourage" by "vicinity" and use "neighbourhood" (voisinage) in its usual sense.

(3) Obviously, both the definition (i) and the notion of $V$-net can be stated for uniform spaces which are not necessarily functional spaces. 
(v) that a family $H$ is equally almost smooth if, to every $V$, there corresponds $U$ and a non-negative integer $n(V)$, such that all but $n(V)$ members of $H$ satisfy

$$
h(U(x)) \subset V(h(x))
$$

(if $n(V) \equiv 0$, the family $H$ is uniformly equicontinuous);

(vi) that a family $H$ has scattered smoothness if, to every $V$, there corresponds $U$, and an infinity of distinct members of $H$, for which (2) is true.

2. Immediate consequences of the definitions. Classical arguments of analysis yield, at once, a number of elementary propositions which we now state (without proofs).

I. Let $h_{1}$ and $h_{2}$ be two mappings which differ by less than $V$ and let $h_{1}$ be $V$-continuous. Then $h_{2}$ is ${ }^{3} V$-continuous.

II. Let (1) be a V-net for the family $H$ and let the elements of (1) be $V$-continuous. Then, there exists $U$ such that

$$
h(U(x)) \subset{ }^{3} V(h(x)),
$$

for all $h[\in H]$ and all $x[\in E]$.

Propositions III, IV, and V require that the structure of $F$ be separated.

III. Let $H$ be almost smooth. Then every point of accumulation of $H$ is uniformly continuous.

IV. Let $H$ be equally almost smooth. Then its derived set $H^{\prime}$ is uniformly equicontinuous.

V. Let $H$ be an infinite family of mappings; if $H^{\prime}$ contains some uniformly continuous mapping, then $H$ has scattered smoothness.

By analogy with metric spaces we say that a uniform space $F$ is totally bounded $\left({ }^{4}\right)$ if, for every $V$, there exists, in $F, V$-nets $\left({ }^{5}\right)$ of $F$. The following lemma is implicitly contained in Gottschalk's proof of Ascoli's Theorem.

LeMma 1. Let $E$ and $F$ be two totally bounded uniform spaces and let $V$ be a symmetrical vicinity of $F$. Let there exist $U$ such that (2) is true for every mapping of a family $H$. Then, $H$ contains $a^{3} V$-net of itself.

From Lemma 1 and II, we deduce a new version of

Theorem of Ascoli-GotTschalk. Let $E$ and $F$ be totally bounded uniform spaces. If the family $H$ is equally almost smooth, then the subspace $H\left(\right.$ of $\left.\mathcal{F}_{u}(E, F)\right)$ is totally bounded. Conversely, if $H$ is almost smooth and totally bounded, then the family $H$ is equally almost smooth.

3. Results of this paper for mappings of $E$ onto itself $\left(^{6}\right)$. We now take $E=F$ to be totally bounded. Let $H$ be a family of mappings of $E$ onto the

(4) Cf. Alexandroff-Hopf [1, p. 104], Gottschalk [4, p. 635], and Bourbaki [2, chap. 2, p. 111].

(s) Cf. Footnote 3.

( $)$ This paper continues a previous investigation of the author [3]. 
whole of $E$. We consider $H$ as a point set of the functional space $\mathcal{F}_{u}(E, E)$ and form its derived set $H^{\prime}$ (that is, the set of points of accumulation of $H$ ). We also define:

$H_{c}^{\prime}$, the set of all uniformly continuous mappings of $H^{\prime}$;

$H_{c}^{*}$, the set of all one-to-one mappings of $H_{c}^{\prime}$.

If $E$ is separated, and $H$ is almost smooth, it follows, from III, that $H_{c}^{\prime}=H^{\prime}$.

If all elements of $H$ are one-to-one, we denote by $H^{-1}$ the set of all the inverse mappings of $H$; we also form $\left(H^{-1}\right)^{\prime},\left(H^{-1}\right)_{c}^{\prime},\left(H^{-1}\right)_{c}^{*}$, the respective analogues of $H^{\prime}, H_{c}^{\prime}, H_{c}^{*}$.

The product of two mappings, $f g=h$, is the mapping $f(g(x))=h(x)$; $e(x)[\equiv x]$ is the identical mapping.

We sometimes take $H$ to be a semi-group, that is, closed under the above multiplication (we do not assume $e \in H$ ).

Definitions. We say that a family $H$ is:

Iterative $\left({ }^{7}\right)$ if, in each infinite subset $\{g\}$, of $H$, there exist two distinct mappings $g_{1}$ and $g_{2}$ such that the equation

$$
u g_{1}=g_{2}
$$

admits of a solution $u$, in $H$.

Strongly iterative if, to every $h[\in H]$, there corresponds, in every infinite subset $\{g\}$, of $H$, a mapping $g$ such that

$$
u h=g
$$

admits of a solution $u$, in $H$.

Obviously, a strongly iterative family is also iterative; on the other hand, it is not difficult to construct iterative families which are not strongly iterative $\left({ }^{8}\right)$.

Our definitions yield the following theorem.

THEOREM 1. Let $E$ be a totally bounded uniform space and $H$ an iterative family of mappings of $E$ onto itself. If $H$ has scattered smoothness, then $e \in H^{\prime}$. Moreover, if $H$ is also a semi-group, then $(e \cup H) \subset H^{\prime}$.

Corollary 1. If $E$ is separated and $H$ is iterative, then $e \in H^{\prime}$ if, and only if, $H^{\prime}$ has scattered smoothness.

COROLLARY 2. If $E$ is separated and $H$ is an iterative semi-group of scattered smoothness, then $H_{c}^{\prime}$ is a semi-group with a unit element.

If $H$ is almost smooth, every element of $H$ is uniformly continuous.

If $H$ is commutative, so is $H_{c}^{\prime}$.

(7) This terminology hints at the fact that the successive iterates of a mapping form a strongly interative semi-group.

( ${ }^{8}$ ) Let $f$ and $g$ be two mappings which commute and such that $f^{m} g^{n}=f^{p} g^{q}$ implies $m=p$ and $n=q$. The set of all mappings of the form $f^{m} g^{n}(0 \leqq m \leqq n)$ is iterative but not strongly iterative. 
For strongly iterative families, Theorem 1 and Corollary 2 can be substantially sharpened. We obtain:

THEOREM 2. Let $E$ be a totally bounded, separated, uniform space. If $H$ is strongly iterative and has scattered smoothness, then every member of $H$ is oneto-one and $H^{-1} \subset H^{\prime}$.

Corollary 3. If, in addition to the assumptions of Theorem 2, $H$ is commutative, then

$$
\left(H^{-1} \cup e \cup H\right) \subset H^{\prime} \quad \text { and } \quad H^{\prime}=\left(H^{-1}\right)^{\prime} .
$$

Corollary 4. If, in addition to the assumptions of Theorem 2, $E$ is compact and $H$ is almost smooth, then

$$
\left(H^{-1} \cup e \cup H\right) \subset H^{\prime} \text { and } H^{\prime}=\left(H^{-1}\right)^{\prime} .
$$

CoROllaRy 5. If $E$ is compact and $H$ is a strongly iterative semi-group of scattered smoothness, then $H_{c}^{*}=\left(H^{-1}\right)_{c}^{*}$ is a group.

If $H$ is commutative and almost smooth, the "schlicht" character of the mappings of $H$ is not lost by a passage to the limit. Corollary 5 then takes the following more satisfactory $\left({ }^{9}\right)$ form.

CoRollary 6. Let $E$ be compact and let $H$ be a commutative, strongly iterative, semi-group. If $H$ is almost smooth and has scattered smoothness, then $H^{\prime}=\left(H^{-1}\right)^{\prime}$ is a group.

THEOREM 3. If $E$ is compact and $H$ is an infinite strongly iterative semigroup, equally almost smooth, then $H^{\prime}=\left(H^{-1}\right)^{\prime}$ is a group and its elements are uniformly equicontinuous.

4. Proof of Theorem 1 and its corollaries. Given $V$, take a symmetrical vicinity $\left({ }^{10}\right) W$, such that ${ }^{6} W \subset V$. By the definition of scattered smoothness, there exist $U$, and an infinite sequence

$$
h_{1}, h_{2}, \cdots, h_{n}, \cdots
$$

of elements of $H$, such that

$$
h_{n}(U(x)) \subset W\left(h_{n}(x)\right),
$$

for every $n$.

(9) In the noncommutative case, the difficulty in improving on Corollary 5 arises from the fact that $H_{c}^{\prime} \neq H_{c}^{*}$. Examples of this behaviour are easy to obtain. Let $H=\{h\}$ be the set of all real, strictly increasing, continuous functions defined for $0 \leqq x \leqq 1$, and such that $h(0)=0$, $h(1)=1$. Considered as a set of mappings of the interval $[0,1]$ onto itself, $H$ is a group and, a fortiori, a strongly iterative semi-group. Also, $H$ has scattered smoothness; however, $H_{c}^{\prime} \neq H_{c}^{*}$.

${ }^{(10)}$ From now on, the symbol $W$ denotes a symmetrical vicinity such that some conveniently chosen iterate of $W$ is contained in a given $V$. Moreover, our notation is such that every relation of the form $a \subset V(b)$ also implies $b \subset V(a)$. 
Applying Lemma 1 to (3), we see that there exist, in (3), a finite number of elements which form a ${ }^{3} W$-net of (3). Hence, there exists an infinite set $\{g\}$, of elements of (3), which differ, by less than ${ }^{3} W$, from some particular element of the ${ }^{3} W$-net. Obviously, any two mappings of $\{g\}$ differ by less than ${ }^{6} W$ and, therefore, by less than $V$. Now $H$ being iterative, there exist $g_{1}$ and $g_{2}$, in $\{g\}$, such that

$$
u g_{1}=g_{2} \quad\left[u \in H, g_{1} \neq g_{2}\right] .
$$

Given $x$, let $y$ be some solution of the equation $g_{1}(y)=x$. Then

$$
g_{1}(y) \subset V\left(g_{2}(y)\right)=V\left(u\left(g_{1}(y)\right)\right)
$$

can be written as

$$
x=e(x) \subset V(u(x)) .
$$

Replacing $x$ by $h(x)$, in (4), we obtain

$$
h(x) \subset V(u(h(x))) \text {. }
$$

As $V$ is arbitrary and $u \neq e,(4)$ proves the first part of Theorem 1, and (5) the second.

Corollary 1 is an immediate consequence of V. In order to prove Corollary 2 , it is sufficient to observe that it is a combination of Theorem 1 and the following lemma, which we state without proof.

LEMMA 2. Let $E$ be a separated uniform space and $H$ a semi-group of mappings of $E$ onto itself. If $H_{c}^{\prime}$ is not empty, it is a semi-group. If $H$ is commutative, so is $H_{c}^{\prime}$.

The proof of this lemma requires only simple continuity arguments; it makes no use of the assumptions of Theorem 1 and does not require that $E$ be totally bounded.

5. Proof of Theorem 2 and Corollaries 3, 4 and 5. Take $V$ arbitrary and $W$ symmetrical, such that ${ }^{2} W \subset V$. As $E$ is separated, Theorem 1 implies the existence of an infinite set $\{g\}$, of distinct mappings of $H$, which differ from $e$ by less than $W$. Let $h$ be any element of $H$; as $H$ is strongly iterative, we can find a $g$ such that

$$
u h=g
$$

$$
[u \in H]
$$

Let $y_{1}$ and $y_{2}$ be solutions of the equation $h(y)=x$. Then

$$
y_{i}=e\left(y_{i}\right) \subset W\left(g\left(y_{i}\right)\right)=W\left(u\left(h\left(y_{i}\right)\right)\right)=W(u(x)) \quad(i=1,2),
$$

from which we deduce

$$
y_{1} \subset{ }^{2} W\left(y_{2}\right) \subset V\left(y_{2}\right) .
$$

As the structure of $E$ is separated and $V$ is arbitrary, this implies $y_{1}=y_{2}$. 
Hence $h^{-1}(x)$ is single-valued and (6) can be written as

$$
h^{-1}(x) \subset W(u(x))
$$

Obviously, we can choose $u \neq h^{-1}$, therefore $H^{-1} \subset H^{\prime}$. Corollary 3 is obtained by replacing, in (7), $x$ by $h\left(u^{-1}(x)\right)$; using the commutativity, we obtain

$$
u^{-1}(x) \subset W(h(x))
$$

As $u^{-1} \in H^{\prime},(8)$ implies $h \subset H^{\prime}$.

To prove $H^{\prime}=\left(H^{-1}\right)^{\prime}$, we note that (8) also implies $H \subset\left(H^{-1}\right)^{\prime}$ and therefore,

$$
H^{\prime} \subset\left(H^{-1}\right)^{\prime \prime} \subset\left(H^{-1}\right)^{\prime} .
$$

On the other hand, by Theorem $2, H^{-1} \subset H^{\prime}$ and, therefore,

$$
\left(H^{-1}\right)^{\prime} \subset H^{\prime \prime} \subset H^{\prime} \text {. }
$$

The proof of Corollary 3 is now complete.

For both Corollaries 4 and 5 we need the following lemma.

Lemma 3. If, in addition to the assumptions of Theorem 2, $E$ is compact, $f \in H_{c}^{*}$ implies $f^{-1} \in H_{c}^{*}$.

Proof. Take $V$ arbitrary and $W$ symmetrical, such that ${ }^{2} W \subset V$. Compactness of $E$ implies uniform continuity of $f^{-1}$ so that we can choose $U$ such that

$$
f^{-1}(U(x)) \subset W\left(f^{-1}(x)\right) ;
$$

we then take $h[\in H]$ such that

$$
f(x) \subset U(h(x))
$$

Then, from (10) and (9),

$$
x=f^{-1}(f(x)) \subset f^{-1}(U(h(x))) \subset W\left(f^{-1}(h(x))\right) .
$$

Replacing $x$ by $h^{-1}(x)$, we obtain

$$
h^{-1}(x) \subset W\left(f^{-1}(x)\right) .
$$

By Theorem 2 there exists a mapping $u(x)$, of $H$, such that

$$
u(x) \subset W\left(h^{-1}(x)\right)
$$

From (12) and (11) we deduce

$$
u(x) \subset{ }^{2} W\left(f^{-1}(x)\right) \subset V\left(f^{-1}(x)\right) .
$$

Hence $f^{-1} \in H^{\prime}$ and, therefore, $f^{-1} \in H_{c}^{*}$.

Proof of Corollary 4. By Theorem 2, any $h[\in H]$ is one-to-one and $h^{-1} \in H^{\prime}$. As $H$ is almost smooth, III shows that $h^{-1}$ is uniformly continuous. 
Hence, $h^{-1} \subset H_{c}^{*}$ and, by Lemma $3, h \subset H_{c}^{*} \subset H^{\prime}$. We still have to show

$$
H^{\prime}=\left(H^{-1}\right)^{\prime} \text {. }
$$

Commutativity, which, in the proof of Corollary 3 , enabled us to pass from (7) to (8), is now replaced by a continuity argument. The mapping $h$ being uniformly continuous, we can, given $V$, choose a symmetrical $W[\subset V]$, and $U$, such that

$$
h(U(x)) \subset W(h(x))
$$

As $h^{-1} \in H^{\prime}$, there exist, in $H$, two distinct mappings $u_{1}$ and $u_{2}$ such that

$$
h^{-1}(x) \subset U\left(u_{i}(x)\right) \quad(i=1,2) .
$$

Then

$$
\begin{array}{cc}
x=h\left(h^{-1}(x)\right) \subset h\left(U\left(u_{i}(x)\right)\right) \subset W\left(h\left(u_{i}(x)\right)\right) & (i=1,2), \\
u_{i}^{-1}(x) \subset W(h(x)) & (i=1,2) .
\end{array}
$$

One at least of the two functions $u_{1}^{-1}, u_{2}^{-1}$ is not identical with $h$. This implies $H \subset\left(H^{-1}\right)^{\prime}$ and, therefore, (13) (cf. the analogous proof of Corollary 3).

Under the assumptions of Corollary 5, both Corollary 2 and Lemma 3 are valid, so that $H_{c}^{*}$ is a group. Obviously, (13) implies $H_{c}^{*}=\left(H^{-1}\right)_{c}^{*}$

6. Proof of Corollary 6. Consider any $f \in H^{\prime}$. Given $V$, let $V_{f}$ be the set of all mappings of $H$ which differ from $f$ by less than $V$; this set is not empty. Now consider the set $\left\{V_{f}\right\}$ where $V$ runs through all vicinities of $E$. Obviously, $\left\{V_{f}\right\}$ is the base of a filter and, as any two elements of $V_{f}$ differ by less than ${ }^{2} V$, the filter with base $\left\{V_{f}\right\}$ is a Cauchy filter. We define $\tilde{V}_{f}$ as the set of all the inverse mappings of $V_{f}$ and verify that any two elements of $\tilde{V}_{f}$ differ by less than ${ }^{2} V$. Let $u$ and $v$ be any two mappings of $V_{f}$; then

$$
u(x) \subset{ }^{2} V(v(x))
$$

and, by Theorem $2, u^{-1}$ and $v^{-1}$ exist.

Replacing, in (14), $x$ by $u^{-1}\left(v^{-1}(x)\right)$, and using the commutativity, we obtain

$$
v^{-1}(x) \subset{ }^{2} V\left(u^{-1}(x)\right) .
$$

Hence $\left\{\tilde{V}_{f}\right\}$ is the base of a Cauchy filter and, as $E$ is compact, this filter is convergent. Let $l(x)$ be its limit. As $H$ is almost smooth and $E$ is compact, both $f$ and $l$ are uniformly continuous (as points of accumulation of continuous functions). We now show that

$$
l(f(x))=f(l(x))=e(x) .
$$

Given $V$, take $W$ symmetrical and such that ${ }^{4} W \subset V$. The uniform con- 
tinuity of $f$ and $l$ enables us to find a symmetric $U[\subset W]$, such that the relations

$$
f(U(x)) \subset W(f(x)), \quad l(U(x)) \subset W(l(x)),
$$

are simultaneously true.

We then choose $w[\in H]$ such that

$$
w(x) \subset U(f(x)), \quad w^{-1}(x) \subset U(l(x)),
$$

are both true.

Then, from (15), (16), and Proposition I, we deduce

$$
w(U(x)) \subset{ }^{3} W(w(x)), \quad w^{-1}(U(x)) \subset{ }^{3} W\left(w^{-1}(x)\right) .
$$

From (16) and (17),

$$
\begin{aligned}
& e(x)=w^{-1}(w(x)) \subset w^{-1}(U(f(x))) \subset{ }^{3} W\left(w^{-1}(f(x))\right), \\
& e(x)=w\left(w^{-1}(x)\right) \subset w(U(l(x))) \subset{ }^{3} W(w(l(x))) .
\end{aligned}
$$

The relations (16) also imply

$$
w(l(x)) \subset W(f(l(x))), \quad w^{-1}(f(x)) \subset W(l(f(x))) .
$$

Substituting (19) into (18), we obtain

$$
e(x) \subset{ }^{4} W(l(f(x))), \quad e(x) \subset{ }^{4} W(f(l(x))) ;
$$

this proves $l=f^{-1}$. Now, by construction, $l \subset\left(H^{-1}\right)^{\prime}$ and, by Corollary 4, $H^{\prime}=\left(H^{-1}\right)^{\prime}$; hence $f^{-1} \subset H^{\prime}\left[=H_{c}^{\prime}\right]$. This, together with Corollary 2, shows that $H^{\prime}$ is a group.

7. Proof of Theorem 3. In all but one step, the proof of Theorem 3 coincides with the proof of Corollary 6. Again we define the Cauchy filter with base $\left\{V_{f}\right\}$ and have to show that $\left\{\tilde{V}_{f}\right\}$ is the base of a Cauchy filter. Our former argument, based on commutativity, should be replaced by an argument involving equicontinuity.

Given $V$, choose $U$ such that

$$
f(U(x)) \subset V(f(x))
$$

for every $f \in H^{\prime}$. Let $T$ be a symmetrical vicinity such that ${ }^{2} T \subset U$ and let $u$ and $v$ be any two elements of $T_{f}$. Then

$$
u(x) \subset{ }^{2} T(v(x)) \subset U(v(x)) \text {. }
$$

Now, $u^{-1}$ being an element of $H^{\prime},(21)$ and (20) imply

$$
\begin{gathered}
e(x)=u^{-1}(u(x)) \subset u^{-1}(U(v(x))) \subset V\left(u^{-1}(v(x))\right), \\
v^{-1}(x) \subset V\left(u^{-1}(x)\right) .
\end{gathered}
$$

Hence any two elements of $\tilde{T}_{f}$ differ by less than ${ }^{2} V$, and $\left\{\tilde{V}_{f}\right\}$ is the base of 
a Cauchy filter. From this point on, the proof of Theorem 3 coincides with that of Corollary 4.

\section{REFERENCES}

1. P. Alexandroff and H. Hopf, Topologie, Berlin Springer, 1935.

2. N. Bourbaki, Topologie gênérale, Hermann, Paris, 1940-1949, chaps. 1-10.

3. A. Edrei, On iteration of mappings of a metric space onto itself. In print: J.London Math. Soc.

4. W. H. Gottschalk, Almost periodicity, equicontinuity and total boundedness, Bull. Amer. Math. Soc. vol. 52 (1946) pp. 633-636.

UNIVERSITY OF SASKaTCHEWAN,

Saskatoon, Sask., Canada. 